\title{
Hepatitis A seroprevalence in homosexual and heterosexual men
}

\author{
R Nandwani, S Caswell, F Boag, A G Lawrence, J C Coleman
}

\begin{abstract}
Objective-To determine the seroprevalence of hepatitis $A$ antibodies in homosexual and heterosexual males attending a genitourinary medicine (GUM) clinic. Design-Prospective study of male patients recruited from a GUM clinic during a 10 week period in 1993.

Setting-Central London outpatient GUM department at Chelsea and Westminster Hospital.

Subjects-255 patients were recruited, comprising 185 homosexual and 70 heterosexual males. Ninety two men were known to be HIV-positive, of whom 89 were homosexual.

Main outcome measures-Serum samples were screened for both IgM and IgG antibodies to hepatitis $A$ by enzyme linked immunoassay. Results were matched to an anonymously completed questionnaire.
\end{abstract}

Results -81 of the 255 subjects $(31 \cdot 8 \%)$ had been exposed to hepatitis $A$, two of whom were IgM positive. There were similar hepatitis A seroprevalence rates in homosexual (32.4\%) and heterosexual men $(30 \cdot 0 \%)$. Although $48 \cdot 1 \%$ of the homosexual men were known to be HIVpositive, compared with $4 \cdot 3 \%$ of the heterosexuals. Hepatitis A seroprevalence remained comparable in both groups after patients with known HIV infection were excluded from the analysis. $11 \cdot 4 \%$ of the heterosexual men admitted to oralanal sexual contact compared with $62 \cdot 2 \%$ of the homosexual men. This sexual practice was not associated with antibodies or a past history of hepatitis $A$ exposure.

Conclusions-There was no detectable difference in hepatitis $A$ seroprevalence between male homosexual and heterosexual GUM clinic attenders, despite a much higher level of oral-anal sexual activity among the homosexual population.

Department of

Medicine

R Nandwani

F Boag

A G Lawrence

Department of

Medical Virology

S Caswell

J C Coleman

Address correspondence to: Dr Rak Nandwani, Claude Nicol Centre, Royal Susse County Hospital, Eastern Road, Brighton BN2 5BE UK.

Accepted for publication 4 May 1994
(Genitourin Med 1994;70:325-328)

\section{Introduction}

The incidence of acute hepatitis $A$ virus (HAV) infection in England and Wales has declined from a peak of 7545 laboratory reports in 1990 to 6762 in 1992 (unpublished Communicable Disease Surveillance Centre data to 30 June 1993). It is unclear whether sexual behaviour is a significant factor in the transmission of HAV, even though the epidemiological association between sexually transmitted hepatitis B infection and homosexual men in the UK has been well documented. ${ }^{1-3}$ As HAV is transmitted by the faecal-oral route, homosexual men have been thought to be at increased risk by oral-anal sexual contact ("rimming") 4 and it is postulated that sexually transmitted outbreaks have occurred in this population. ${ }^{56}$ However, on closer examination, it appears that in many instances the spread of HAV was significantly related to occupational factors such as airline travel, medical care and food handling, ${ }^{7}$ and that homosexual men were simply an easily identifiable population in the community, who are commonly employed in risk professions for hepatitis A. ${ }^{5}$

Since the onset of the HIV epidemic, "safer sex" practices which avoid unprotected penetrative anal intercourse have been promoted, possibly increasing the prevalence of oral-anal sexual contact ${ }^{8}$ and therefore HAV infection in both homosexual ${ }^{9}$ and heterosexual men. A formaldehyde inactivated hepatitis $A$ vaccine (Havrix, SmithKline Beecham) is now available, but it is unclear whether homosexual men should routinely be offered immunisation. ${ }^{10}$

The aim of our study was to determine the seroprevalence of HAV infection in male genitourinary (GUM) medicine clinic attenders and to examine its relationship to several factors including sexual orientation, HIV status and sexual behaviour including oral-anal sexual contact in order to assist the formulation of future strategies regarding the administration of hepatitis A vaccine.

\section{Subjects and methods}

Study population

The study population was recruited over a ten week period in 1993 from male patients attending the genitourinary outpatient clinic at St Stephen's clinic (which is part of the Chelsea \& Westminster hospital). Volunteers were given an information sheet about hepatitis A and were asked to complete an anonymous questionnaire which collected data on factors including: occupation, country of birth, travel, sexual behaviour; and previous hepatitis exposure. We limited our questions on sexual activity to the preceding year as we noted that volunteers experienced difficulty in accurately recalling the precise nature of practices more than 12 months previously. Many of the participants were new clinic attendees 
who were having blood tests for syphilis, HIV or for other reasons. A total of 556 new male patients attended the clinic during the recruitment period. Subjects were excluded from the study if they were aged below 16 years as it was considered that they were unable to give valid informed consent. Local ethical committee approval was granted prior to the start of the study.

\section{Serum testing}

Blood was taken and matched to the questionnaire using a numbered label. Serum was stored and subsequently tested for hepatitis A using a competitive enzyme linked immunoassay method (Launch Biokit) for antibodies against both hepatitis A IgM and IgG. Four samples without accompanying questionnaires were discarded.

\section{Statistical analysis}

A one-tailed chi square test was used to compare differences between the hepatitis A seropositive and seronegative groups.

\section{Results}

Bisexual men have been included with homosexual men in all analyses. Data was analysed from 255 serum samples with completed questionnaires. This sample comprised 185 homosexual and 70 heterosexual men. The vast majority ( 211 out of 255 ) had taken HIV tests. Of these, 92 were known to be HIVpositive (of whom 89 were homosexual men) and 119 were HIV-negative. The HIV status was unknown in only 44 subjects. There was no significant difference in the mean age, country of birth or recent travel history outside the UK between the homosexual and

Table 1 Demographic characteristics

\begin{tabular}{lll}
\hline & Homosexual & Heterosexual \\
\hline Total number & 185 & 70 \\
Mean age (years) & $32 \cdot 6$ & $32 \cdot 2$ \\
Born in UK (\%) & $139(75 \cdot 1 \%)$ & $53(75 \cdot 7 \%)$ \\
Travel abroad within & $144(77 \cdot 8 \%)$ & $56(80 \cdot 0 \%)$ \\
$\quad$ previous year (\%) & & \\
Known HIV positive (\%) & $89(48 \cdot 1 \%)$ & $3(4 \cdot 3 \%)$ \\
\hline
\end{tabular}

Table 2 Anti-HAV seroprevalence (all male patients)

\begin{tabular}{lll}
\hline & Homosexual & Heterosexual \\
\hline Anti-HAV positive & 60 & 21 \\
$\%$ seroprevalence & $32 \cdot 4 \%$ & $30 \cdot 0 \%$ \\
$95 \%$ CI & 26.0 to $39 \cdot 6 \%$ & $20 \cdot 4$ to $41 \cdot 8 \%$
\end{tabular}

Table 3 Anti-HAV seroprevalence after exclusion of known HIV-positives

\begin{tabular}{lll}
\hline & Homosexual & Heterosexual \\
\hline Not known HIV-infected & 96 & 67 \\
Anti-HAV positive & 25 & 18 \\
$\%$ seroprevalence & $26 \cdot 0 \%$ & $26.9 \%$ \\
$95 \%$ CI & $18 \cdot 1$ to $35 \cdot 8 \%$ & $17 \cdot 6$ to $38 \cdot 8 \%$ \\
\hline
\end{tabular}

Table 4 Oral-anal sexual contact ("rimming") in 185 homosexual male clinic attenders

\begin{tabular}{lll}
\hline & Rimming within 1 year & No rimming within 1 year \\
\hline Total number & 115 & 70 \\
Anti-HAV positive (\%) & $38(33 \cdot 0 \%)$ & $22(31 \cdot 4 \%)$ \\
$95 \%$ CI & 24.9 to $42 \cdot 2 \%$ & 21.6 to $43 \cdot 2 \%$ \\
\hline
\end{tabular}

heterosexual groups (table 1). The seroprevalence of anti-HAV antibodies by sexual orientation with $95 \%$ confidence intervals is shown in table 2.

Only $2(2.5 \%)$ of the 81 anti-HAV antibody positive serum samples were found to be positive for IgM. Both these patients had clinical signs of hepatitis. One was a homosexual HIV-positive man thought to have been infected by contaminated food. The other was a heterosexual man who attended after unprotected intercourse with a female partner with genital herpes, whose family doctor had previously diagnosed hepatitis A (source unknown). The remainder were all IgG positive, indicating that there had been no epidemic outbreak during the study period.

Of the 92 known HIV-positives, 38 $(41.3 \%)$ had antibodies against HAV compared with 43 of the $163(26.4 \%)$ remaining men not known to be HIV-positive. This was significant $\left(p=0.02, \chi^{2}=5.37\right)$. However, if all patients known to be HIV-positive were excluded from the analysis, the seroprevalence rates remained similar for homosexual and heterosexual men (table 3 ).

There was no significant association found between previous hepatitis $\mathrm{A}$ exposure and sexual practices involving oral-anal contact within the previous year in homosexual men in this study as shown in table 4.

No association was apparent between oralanal sexual contact and hepatitis $A$ in the heterosexual men either, although this activity was much less common than in the homosexual population: eight of the 70 heterosexual men (11.4\%) admitted to participating in oral-anal sexual practices with their female partners within the previous year, compared with $62 \cdot 2 \%$ of the homosexual men.

\section{Discussion}

Owing to difficulties in matching socioeconomic and demographic factors, it is uncertain whether the prevalence of anti-HAV is higher in patients attending GUM clinics compared with the general population; the prevalence of anti-HAV IgG in 863 Foreign Office staff was $39 \%$ in $1989-90,{ }^{11}$ and was noted to have fallen in London blood donors from $47 \%$ in 1977 to $32 \%$ in $1985 .{ }^{12}$

A 1980 GUM clinic study from Nova Scotia $^{13}$ found anti-HAV prevalence rates three times higher in homosexual males $(42 \%)$ and heterosexual males and females (39\%) when compared with blood donors $(13 \%)$ and student nurses $(14 \%),{ }^{14}$ whilst the prevalence rates were similar in heterosexual GUM patients and matched controls in Copenhagen $(28 \%)^{15}$ and New York City $(23 \%) .{ }^{16}$

Furthermore, it is also controversial whether the prevalence of anti-HAV differs between homosexual and heterosexual male GUM clinic attenders. In the absence of a hepatitis A outbreak, it is likely that the majority of infections have been acquired during childhood or by close contact with an infected person and are entirely unrelated to 
sexual activity. A study of 102 homosexual men recruited from a Seattle GUM clinic in the late $1970 \mathrm{~s}^{17}$ found an anti-HAV prevalence rate of $30 \%$ compared with $12 \%$ in matched heterosexuals with recurrent genital herpes simplex. Patient diaries suggested that HAV infection correlated with previous oralanal sexual contact; however, the authors' own HAV incidence data during the study period clearly demonstrated that there had been an epidemic of hepatitis $\mathrm{A}$ in the study population, although it was not established whether this was related to sexual transmission. Danish workers also described differing anti-HAV prevalences of $30 \%$ in homosexuals and $20 \%$ in heterosexuals. ${ }^{18}$ However, the strongest support for our findings come from a 1979 study from a London GUM clinic situated close to our hospital and hence with a similar attendant population, which found similar seroprevalence rates in the two groups, with figures of $49.2 \%$ for homosexuals and $43.8 \%$ for heterosexuals. ${ }^{19}$

The factors prompting oral-anal sexual practices among homosexual men have previously been documented ${ }^{9}$ with a prevalence of up to $76 \% .^{20}$ No previous studies have been published examining corresponding behaviour in heterosexual GUM clinic attenders. Our figure of $11.4 \%$ may be an underestimate, as it is uncertain whether oral-anal sexual contact is becoming more common in this population since the advocacy of "safer sex" activities as regards HIV transmission which avoid penetrative vaginal or anal intercourse. This may also have other implications as it has been postulated that there could be a faecaloral transmitted co-factor involved in the aetiology of Kaposi's sarcoma in AIDS patients. ${ }^{21}$

This is the first study that has examined anti-HAV prevalence in a GUM clinic since the onset of the HIV epidemic and the release of an active hepatitis A vaccine. Despite the conflicting nature of previous published studies, our findings support the view that hepatitis A prevalence rates in homosexual and heterosexual men are broadly comparable. We have been unable to demonstrate any difference in the rates between homosexual and heterosexual GUM clinic attenders with similar demographic characteristics, despite excluding those with known HIV infection.

Although the prevalence of oral-anal sexual practices within the previous year was markedly higher in homosexual men at $62 \cdot 2 \%$ contrasting with $11.4 \%$ in heterosexuals, this has not resulted in a corresponding increase in anti-HAV antibodies as expected. The antiHAV prevalence was almost identical in those homosexual men who had practised oral-anal sexual activities compared to those who had not.

The large percentage of HIV-positive volunteers in our study is explained by the high local prevalence of HIV infection; our district (Kensington, Chelsea \& Westminster) accounted for 2363 of the 7246 AIDS cases reported in England and Wales to the end of June 1993 (PHLS AIDS Centre unpublished quarterly surveillance tables no. 20, June
1993, table 4). It has been hypothesised that there may be some loss of antibodies to HAV in HIV-positive patients with dual infection, but there is no published data to confirm that this occurs. The similar anti-HAV seroprevalence rate after the exclusion of HIV-seropositives does not appear to support this view.

Whilst approximately $80 \%$ of the GUM clinic attenders had travelled outside the UK within the preceding year, there is no evidence that this population should routinely be offered hepatitis A vaccination solely to prevent sexual transmission. However, there may well be a case for offering active hepatitis $A$ immunisation to non-immune travellers in the GUM clinic setting simply because this is a suitable point of delivery of vaccine to individuals who travel extensively. The costs of such a strategy would require careful evaluation.

To conclude, quantitating anti-HAV seroprevalence is extremely difficult owing to confounding demographic factors and recruitment of sufficient sample size, but our large study of similar populations of homosexual and heterosexual men attending a central London GUM clinic has demonstrated that the anti-HAV prevalence is similar in both groups, and is not significantly altered by the exclusion of individuals with known HIV infection. Although we have shown that oralanal sexual activity is not exclusively restricted to homosexual men, this does not appear to be a major route of transmission for hepatitis $\mathrm{A}$ in the context of cumulative lifetime risk. Further studies are required at other centres to assess the local risk of HAV infection which may be influenced by geographical factors as well as a more detailed assessment of sexual behaviour by the attendant population.

We thank David Cutland and the staff of the John Hunter Clinic for their assistance, and Dr K MacRae, reader in medical statistics, for performing the data analysis. Salmah Zain helped with the serum testing. The PHLS Communicable Disease Surveillance Centre figures were kindly supplied by Dr J Heptonstall.

1 Coleman JC, Waugh M, Dayton R. Hepatitis B antigen and antibody in a male homosexual population. $B r f$ Venereal Dis 1977;53:132-4.

2 Ellis WR, Murray-Lyon IM, Coleman JC, et al. Liver disease among homosexual males. Lancet 1979;i:903-5.

3 Schreeder MT, Thompson SE, Hadler SC, et al. Hepatitis $B$ in homosexual men: Prevalence of infection and fac tors related to transmission. $\mathcal{F}$ Infect Dis 1982;146:7-15.

4 Mindel A, Tedder R. Hepatitis A in homosexuals. BMF 1981;282:1666.

5 Christenson B, Bronstom $\mathrm{CH}$, Bottinger $\mathrm{M}$, et al. An epidemic outbreak of hepatitis $A$ among homosexual men in Stockholm. Am $\mathcal{F}$ Epidemiol 1982;116:599-607.

6 Hoybye G, Skinhoj P, Hentzer B, et al. An epidemic of acute viral hepatitis in male homosexuals: etiology and clinical characteristics. Scand $\mathcal{f}$ Infect Dis 1980;12:241-4.

7 Kosatsky T, Middaugh JP. Linked outbreaks of hepatitis A in homosexual men and in food service patrons and in homosexual men and in food service

$8 \mathrm{CDC}$. Hepatitis A among homosexual men-United States, Canada and Australia. MMWR. 1992;41:155, $161-4$.

9 Kani J, Nandwani R, Gilson RJ, Johnson AM, Maguire HC, Tedder RS. Hepatitis A virus infection amon homosexual men. BMf 1991;302:1399.

10 Atkins M, Zambon M, Watkins P. Should susceptible homosexual men be offered immunisation? BMf 1993 307:562.

11 Tilzey AJ, Banatvala JE. Hepatitis A. BMF 1991;302: 552-3.

12 Vandervelde EM, Millard JM, Parry JV, Mortimer PP Time for action on hepatitis $\mathrm{B}$ immunisation. $B M \mathcal{J}$ 1987;294:301.

13 McFarlane ES, Embil JA, Manuel FR, Thiebaux HJ. Antibodies to hepatitis A antigen in relation to the numAntibodies to hepatitis $A$ antigen in relation to the num-
ber of lifetime sexual partners in patients attending an STD clinic. Br $\mathcal{F}$ Venereal Dis 1981;57:58-61. 
14 McFarlane ES, Embil JA, Manuel FR, Gorelick $M$ Prevalence of antibodies to hepatitis $A$ antigen in patients attending a clinic for sexually transmitted diseases. Sexually Transmitted Diseases 1980;7:87-9.

15 Hentzer B, Skinhoj P, Høybye G, et al. Viral hepatitis in a venereal clinic population: relation to certain risk factors. Scand $\mathcal{F}$ Infect Dis 1980;12:245-9.

16 Szmuness W, Stevens CE, Harley EJ, et al. Hepatitis B vaccine: demonstration of efficacy in a controlled clinical trial in a high-risk population in the United States. $N$ Engl f Med 1980;303:833-41.

17 Corey L, Holmes KK. Sexual transmission of hepatitis A in homosexual men. $N$ Engl $f$ Med 1980;302:435-8.
18 Kryger P, Pederson NS, Mathieson L, Nielsen JO Increased risk of infection with hepatitis $A$ and $B$ viruse in men with a history of syphilis: relation to sexual contacts. F Infect Dis 1982;145:23-6.

19 Coleman JC, Evans BA, Thornton A, Zuckerman AJ Homosexual hepatitis. $\Im$ Infect 1979;1:61-6.

20 Coutinho RA, Albrecht-Van Lent P, Lelie N, et al. Prevalence and incidence of hepatitis $\mathrm{A}$ among male homosexuals. BMF 1983;287:1743-5.

21 Beral V, Bull D, Darby S, et al. Risk of Kaposi's sarcoma and sexual practices associated with faecal contact in homosexual or bisexual men with AIDS. Lancet 1992; 339:632-5. 